\title{
EVALUATION OF MYCOBACTERIAL ANTIGENS IN AN ENZYME-LINKED IMMUNOSORBENT ASSAY (ELISA) FOR THE SERODIAGNOSIS OF TUBERCULOSIS
}

\author{
R. G. Benjamin, S. M. Debanne*, Y. Ma and T. M. Daniel $\dagger$ \\ Departments of Medicine and * Biometry, \\ Case Western Reserve University School of Medicine, \\ Cleveland, Ohio 44106, USA
}

\begin{abstract}
SUMmARY. Five mycobacterial antigens were compared in an enzymelinked immunosorbent assay (ELISA) for the serodiagnosis of tuberculosis. The antigens studied were an unheated sterile culture filtrate of Mycobacterium tuberculosis, tuberculin purified protein derivative (PPD) from $M$. tuberculosis $\left(\mathrm{PPD}_{\mathrm{a}}\right)$, purified cytoplasmic protein antigens 5 and 6 from $M$. tuberculosis, and a PPD prepared from $M$. kansasi $\left(\mathrm{PPD}_{\mathrm{k}}\right)$. Multivariate analysis of variance showed that geometric mean titres obtained with each of the antigens in ELISA were significantly different in tuberculosis patients and in control groups. The covariation of the ELISA results with the five antigens was highly interdependent. Analysis of receiver operating characteristics revealed that the most accurate test was obtained with antigen 5. M. tuberculosis PPD, $M$. tuberculosis antigen 6, and $M$. tuberculosis culture filtrate were, in descending order, less accurate.
\end{abstract}

\section{INTRODUCTION}

Recent studies have shown encouraging results with enzyme-linked immunosorbent assays (ELISA) for the serodiagnosis of tuberculosis (Nassau, Parsons and Johnson, 1976; Tandon et al., 1980; Stroebel et al., 1982; Benjamin and Daniel, 1982; Kalish et al., 1983; Radin, Zeiss and Phair, 1983). There have been some differences in the ELISA methodology, but the major differences have been in the antigens used to detect antibody in patient and control sera. The main types of antigen preparations studied have been highly purified antigens, including proteins and lipids, and, more commonly, antigen mixtures adapted from skin-test preparations.

We recently reported (Benjamin and Daniel, 1982) encouraging preliminary data on the use of purified protein antigen 5 in the serodiagnosis of active pulmonary tuberculosis. The ELISA in that report was both sensitive and specific for the disease when compared with results obtained with a number of control groups. Others have reported good results with nonpurified antigen mixtures (Nassau et al., 1976; Tandon, et al., 1980; Kalish et al., 1983; Radin et al., 1983).

Received 8 Dec. 1983; accepted 22 Jan. 1984.

$\dagger$ Requests for reprints should be addressed to Dr T. M. Daniel. 
In the present study we compared several antigen preparations, including two purified proteins and two purified protein derivatives (PPD). They were tested with sera from patients with active pulmonary tuberculosis and from five control groups. The experimental design enabled determination of sensitivity and specificity.

\section{MATERIALS AND METHODS}

Antigens. Five antigen preparations were studied. Unheated culture filtrate of Mycobacterium tuberculosis strain H37Ra was prepared, as previously described (Daniel and Ferguson, 1970), from cultures grown on totally synthetic medium and used at a concentration of $10 \mu \mathrm{g} / \mathrm{ml}$. Antigens 5 and 6 were prepared from $M$. tuberculosis culture filtrate by immunoabsorbent affinity chromatography, as previously described (Daniel and Anderson, 1977 and 1978), with monospecific goat antisera and used at a concentration of $5 \mu \mathrm{g} / \mathrm{ml}$. PPDs were prepared from heated culture filtrates of $M$. tuberculosis and $M$. kansasi respectively by precipitation with ammonium sulphate at $50 \%$ saturation as previously described (Ma and Daniel, 1983). We designated the product prepared from $M$. tuberculosis $\mathrm{PPD}_{\mathrm{a}}$ and that from $M$. kansasi $\mathrm{PPD}_{\mathrm{k}}$. Each was used at a concentration of $10 \mu \mathrm{g} / \mathrm{ml}$.

ELISA. The assay was performed in round bottomed PVC microtitration plates (Disposable U Plates, Flex Vinyl, Dynatech Laboratories, Alexandria, VA) as described previously (Benjamin and Daniel, 1982). The wells were sensitised with antigen in the presence of glutaraldehyde at a final concentration of $1 \%$. All titrations were performed in duplicate. IgG antibody was measured with a swine anti-human IgG-alkaline phosphatase conjugate (Orion Diagnostika, Helsinki, Finland). Each titration was read against a standard control serum to limit plate-to-plate and day-to-day variation. All sera were coded to eliminate observer bias.

Sera. Sera were obtained from a collection stored at $-70^{\circ} \mathrm{C}$ for various periods up to 15 years. Six subject groups were tested comprising three groups with active disease due to mycobacterial infection, and three control groups.

(i) Pulmonary tuberculosis. A non-homogeneous group of 27 sera from patients in Cleveland hospitals with active pulmonary tuberculosis defined by infiltrates on chest radiographs and identification of $M$. tuberculosis in sputum by smear or culture or both.

(ii) $M$. kansasi infection. Sera from 10 patients seen at University Hospitals of Cleveland or the National Jewish Hospital, Denver, Colorado, with active lung disease caused by $M$. kansasi, defined by isolation of the organism from sputum by culture in the presence of an infiltrate on chest radiograph.

(iii) $M$. intracellulare infection. Sera from 11 patients seen at the National Jewish Hospital with active lung disease caused by $M$. intracellulare, defined by isolation of the organism from sputum culture in the presence of an infiltrate on chest radiograph.

(iv) Sarcoidosis. Sera from 23 patients at University Hospitals of Cleveland with sarcoidosis and no evidence of mycobacterial infection.

(v) PPD-positive controls. Sera from 23 healthy hospital employees who were skin-test positive on testing with intermediate strength PPD.

(iv) PPD-negative controls. Sera from 23 healthy hospital employees who were skin-test negative on testing with intermediate strength PPD.

Serum preparation. All the sera were diluted in preparation for titration at the time of coding and the same diluted sample was used throughout to ensure consistency.

Statistical Methods. A comparison of the efficiencies of the six antigens in the ELISA was performed by measuring the extent to which titres varied amongst the six subject groups. This comparison utilised a two-level, nested (or hierarchical) experimental design. The major classification dichotomy, healthy or diseased with respect to mycobacterial infection, was broken down so that patient groups 1, 2 and 3, with mycobacterial disease, were identified as categories within the group of diseased patients and control groups 4, 5 and 6, without mycobacterial disease, were identified as categories within the group of nondiseased control subjects. This experimental design enabled the testing of differences in responses between health groups and differences between categories within each of the two groups of subjects. It did not allow us to test differences in responses between individual categories in different health groups. 
To gain insight into multivariate antigen relationships and, simultaneously, to test the relevant hypotheses concerning differences among patient groups for each antigen used in the ELISA, multivariate analysis of variance (MANOVA) with two factors (health group and category within group) and six outcome variables (results with the six antigens) was used. To conform with requirements for linearity and homoscedasticity, the data were transformed logarithmically so that the titration end-points were expressed as $\log _{2}$ (titre/10). This transformed variable represented the maximum number of doubling dilutions that gave a positive reaction over the concentration range tested.

Receiver operating characteristic curves were plotted by the method of Metz (1978). Trueand false-positive fractions were calculated for each dilution in the titration series of doubling dilutions.

\section{RESULTS}

The results of the individual serum titrations are presented in figs $1-5$. The geometric mean titres and $95 \%$ confidence limits for the results with each antigen in each group of subjects are included in these figures.

Multivariate considerations of the scores of category within patient and control groups showed that ELISA results with $M$. tuberculosis culture filtrate depended on linear combinations of values of the other four antigens. Consequently they did not contribute additional information and were excluded from subsequent analysis. The

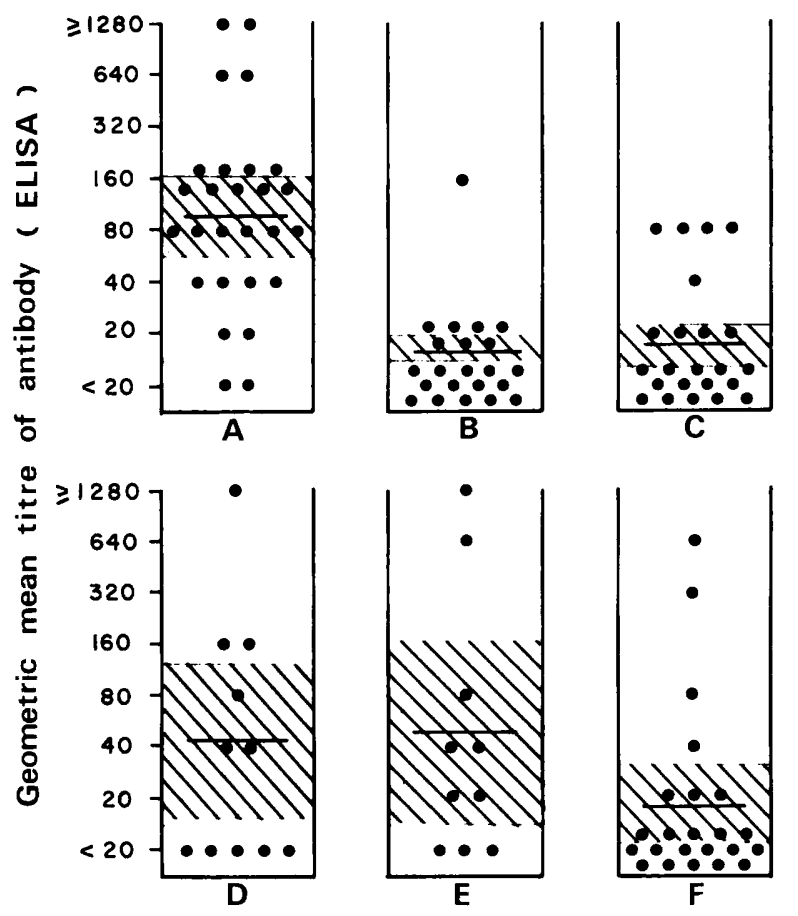

FIG. 1.-ELISA titration results with $M$. tuberculosis antigen 5 in six groups of subjects: A, patients with pulmonary tuberculosis; B, PPD-negative controls; C, PPD-positive controls; D, patients with $M$. intracellulare infection; $\mathrm{E}$, patients with $M$. kansasi infection; F, patients with sarcoidosis. Each result is shown by a single point. Geometric mean titres are indicated by horizontal bars with the cross-hatched area representing the $95 \%$ confidence interval. 

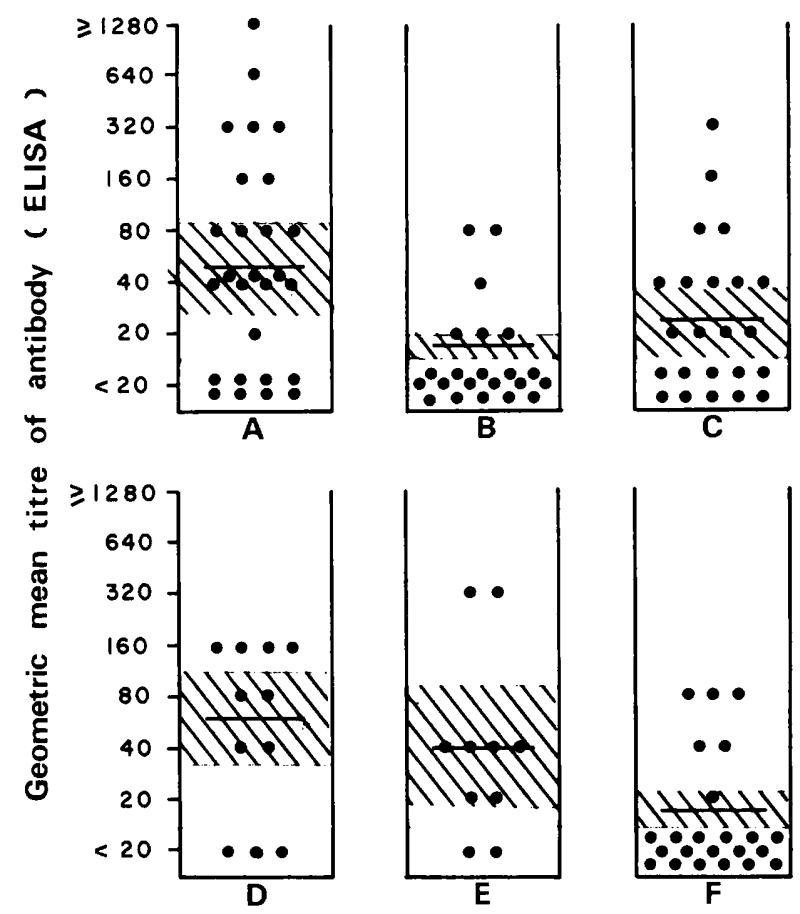

FIG. 2.-ELISA titration results with $M$. tuberculosis antigen 6 in six groups of subjects (see caption to fig. 1).
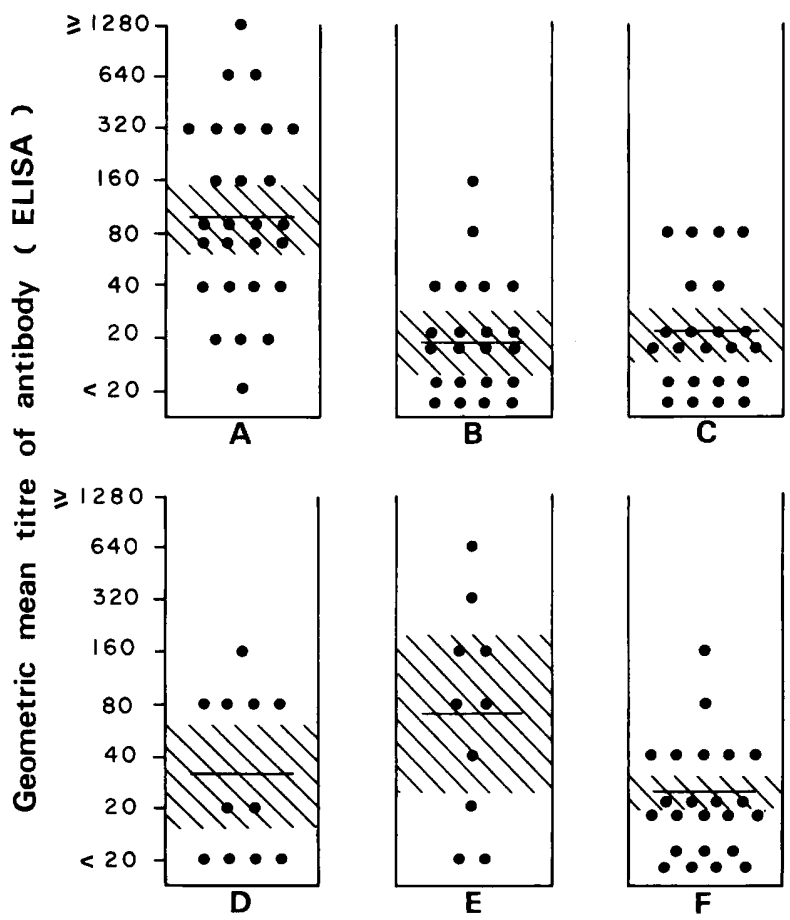

FIg. 3.-ELISA titration results with $\mathrm{PPD}_{\mathrm{a}}$ in six groups of subjects (see caption to fig. 1). 

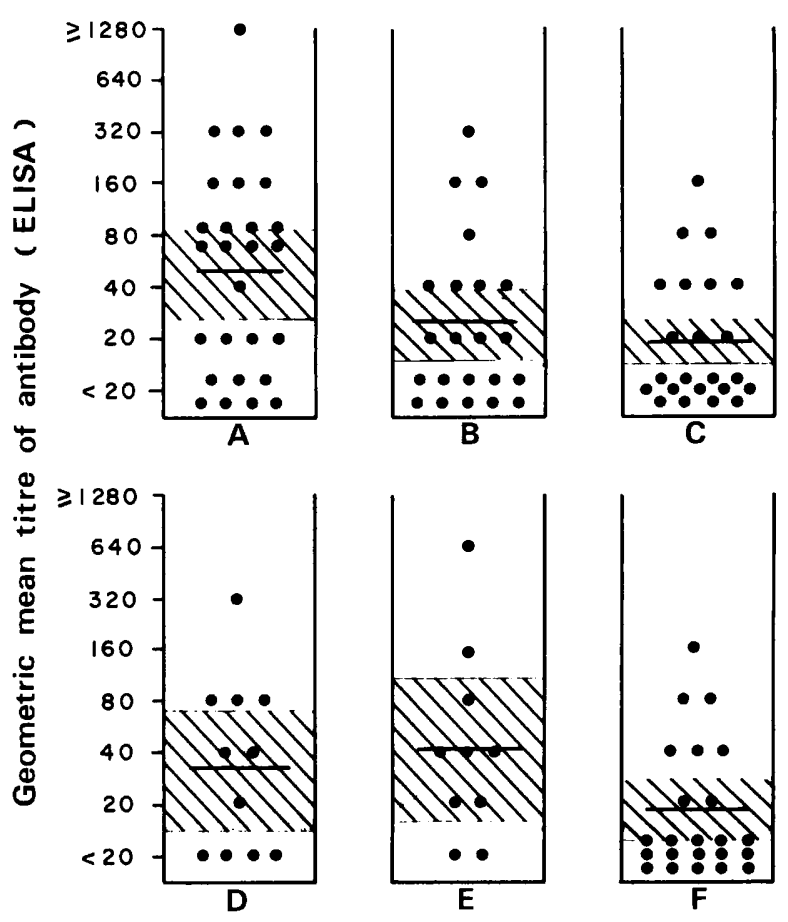

FIG. 4.-ELISA titration results with $M$. tuberculosis unheated culture filtrate in six groups of subjects (see caption to fig. 1).
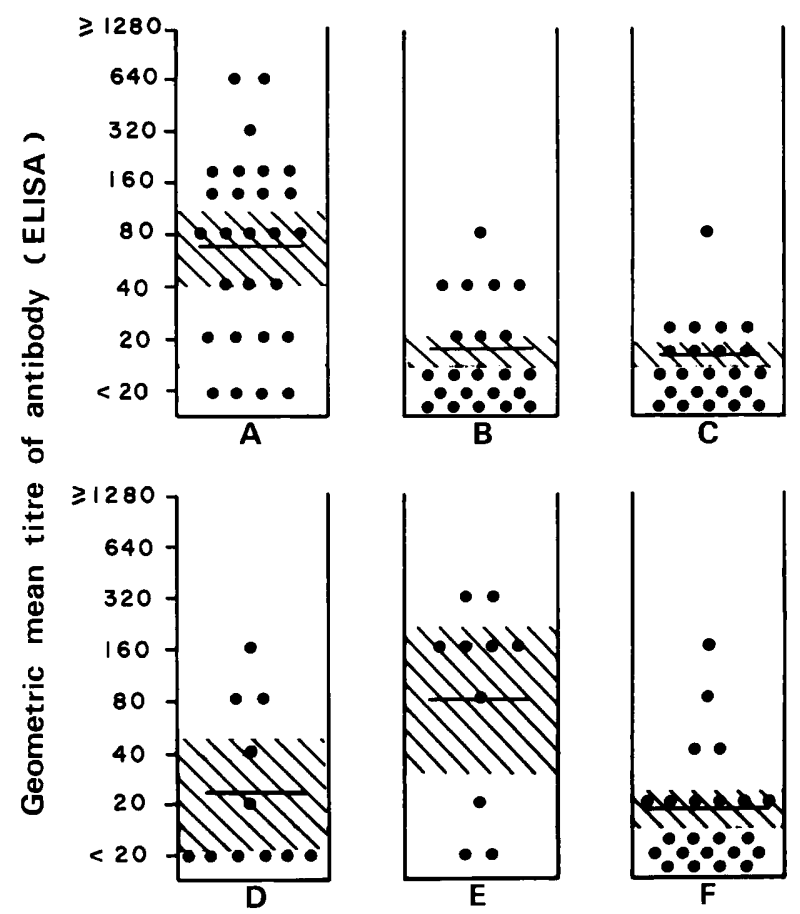

FIG. 5.-ELISA titration results with $P P D_{\mathrm{k}}$ prepared from M. kansasi in six groups of subjects (see caption to fig. 1). 
other outcome variables were not completely independent of each other; however, the amount of information in each remaining variable required that the results with all four be analysed.

Three related statistical tests (Morrison, 1976), Pillai's F, Hotelling's T, and Wilks' $\lambda$ suggested that the manner in which ELISA results with each antigen varied over the spectrum of patient groups was different with the different antigens. Hence, univariate analyses of variance (ANOVA) were performed. These analyses showed that all antigens significantly differentiated between diseased and nondiseased groups. Furthermore, ELISA with $\mathrm{PPD}_{\mathrm{a}}$ and $\mathrm{PPD}_{\mathrm{k}}$ significantly differentiated between individual categories within health groups (table I). Estimates of the effect attributable to each health group and each category within health groups are given in table II. Compound scores based on either the sum of the values obtained with the four antigens

TABLE I

Univariate analyses of variance of ELISA results*

\begin{tabular}{lcccc}
\hline & \multicolumn{3}{c}{ Value obtained with antigens } \\
\cline { 2 - 5 } Sources of variation & Antigen 5 & Antigen 6 & PPD $_{\mathrm{a}}$ & PPD $_{\mathrm{k}}$ \\
\hline Between health groups mean square & $125 \cdot 89$ & $67 \cdot 41$ & $90 \cdot 70$ & $93 \cdot 19$ \\
Between categories within health group mean square & $4 \cdot 25$ & $2 \cdot 24$ & $5 \cdot 98$ & $5 \cdot 95$ \\
$\mathrm{~F}_{1,4}$ Ratio for effect of health group & $29 \cdot 65 \dagger$ & $30.04 \dagger$ & $15 \cdot 17 \dagger$ & $15 \cdot 67 \dagger$ \\
Between categories within health group mean square & $4 \cdot 25$ & $2 \cdot 24$ & $5 \cdot 98$ & $5 \cdot 95$ \\
Within categories mean square & $2 \cdot 78$ & $2 \cdot 29$ & 1.98 & $1 \cdot 80$ \\
$\mathrm{~F}_{4,110}$ Ratio for effect of category within health group & $1.53 \S$ & $0.98 \S$ & $3.02 \dagger$ & $3 \cdot 30 \dagger$ \\
\hline
\end{tabular}

* Results of titrations were expressed as $\log _{2}($ titre/10) for statistical analysis.

† Significant value $(P<\cdot 01) ; \ddagger$ Significant value $(P<\cdot 05) ; \S$ value not significant.

TABLE II

Estimates of effects attributable to health status for antigen 5, antigen $6, P P D_{a}$ and $P P D_{K}$. Predicted scores are given for each antigen within parentheses in the order (antigen 5, antigen $6, P P D_{a}, P P D_{k}$ )

\begin{tabular}{|c|c|c|c|}
\hline Main effect & $\begin{array}{l}\text { Effect of } \\
\text { health group }\end{array}$ & $\begin{array}{l}\text { Effect of } \\
\text { category }\end{array}$ & $\begin{array}{l}\text { Resultant predicted } \\
\text { mean scores* }\end{array}$ \\
\hline \multirow{2}{*}{$\begin{array}{l}\text { Baseline score } \\
(1 \cdot 60,1 \cdot 53,1 \cdot 83,1 \cdot 46)\end{array}$} & $\begin{array}{c}\text { Mycobacterial disease } \\
(+0.93,+0.77,+0 \cdot 77,+0.85)\end{array}$ & $\left\{\begin{array}{c}\text { Tuberculosis } \\
(+0 \cdot 76,+0 \cdot 04,+0 \cdot 77,+0 \cdot 43) \\
M . \text { kansasi disease } \\
(-0 \cdot 32,-0 \cdot 29,+0 \cdot 20,+0 \cdot 69) \\
M . \text { intracellulare disease } \\
(+0 \cdot 44,+0 \cdot 25,-0 \cdot 97,-1 \cdot 12)\end{array}\right.$ & $\begin{array}{l}(3 \cdot 29,2 \cdot 34,3 \cdot 37,2 \cdot 74) \\
(2 \cdot 21,2 \cdot 01,2 \cdot 80,3 \cdot 00) \\
(2 \cdot 97,2 \cdot 55,1 \cdot 63,1 \cdot 19)\end{array}$ \\
\hline & $\begin{array}{c}\text { No mycobacterial disease } \\
(-0.93,-0.77,-0.77,-0.85)\end{array}$ & $\left\{\begin{array}{c}\text { Sarcoidosis } \\
(+0 \cdot 15,-0 \cdot 15,+0 \cdot 07,+0 \cdot 12) \\
\text { PPD-positive controls } \\
(+0 \cdot 02,+0 \cdot 46,+0 \cdot 03,-0 \cdot 14) \\
\text { PPD-negative controls } \\
(-0 \cdot 17,-0 \cdot 31,-0 \cdot 10,+0 \cdot 02)\end{array}\right.$ & $\begin{array}{l}(0.82,0.61,1 \cdot 13,0.75) \\
(0.69,1.22,1.09,0.47) \\
(0.50,0.45,0.96,0.63)\end{array}$ \\
\hline
\end{tabular}

* Predicted mean score for a given health status was obtained by adding the effect of health group and category to the baseline score. 


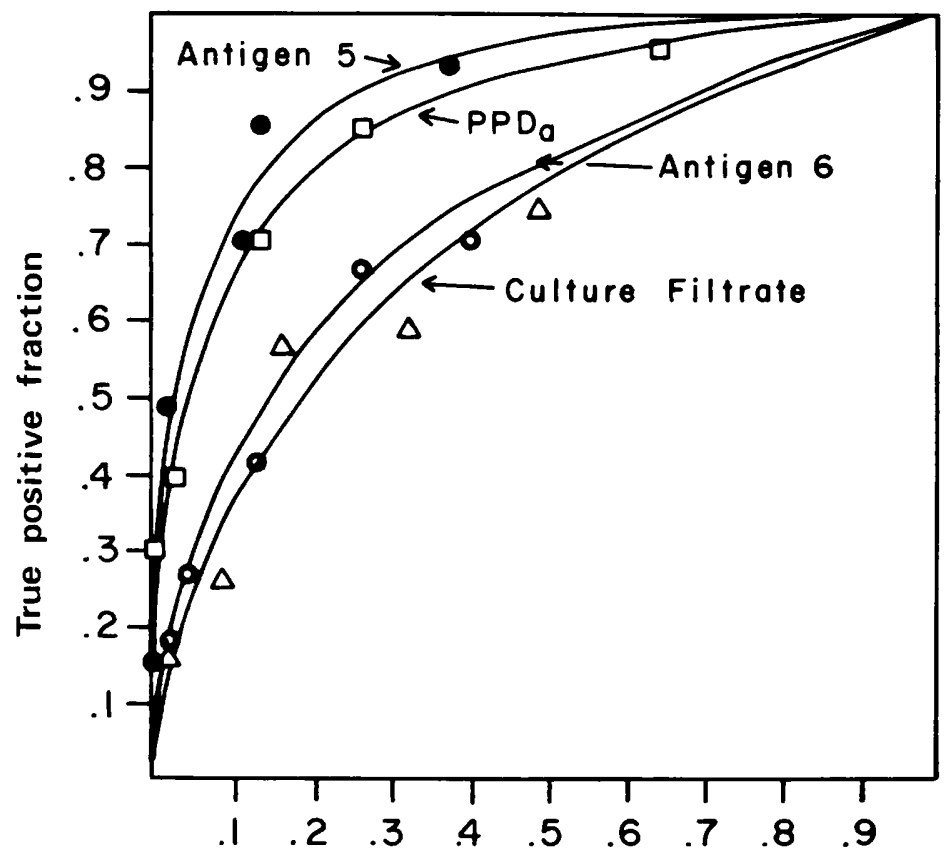

False positive fraction

Fig. 6.-Receiver operating characteristic curves for $M$. tuberculosis antigen $\mathrm{t}(\bullet), M$. tuberculosis antigen 6 $(0), P D_{a}(\square)$, and $M$. tuberculosis unheated culture filtrate $(\Delta)$. The true positive fraction is plotted against the false positive fraction for each antigen at each dilution tested from $<1$ in 20 to 1 in 320 . For each level of sensitivity (true positive fraction), specificity (1-false positive fraction) was greatest with antigen 5 .

or on transformation of paired combinations of them failed to yield significant new information.

Receiver operating characteristic curves are shown in fig. 6 for culture filtrate, $\mathrm{PPD}_{\mathrm{a}}$, antigen 5 and antigen 6 . These curves are based on results obtained with sera from tuberculosis patients compared with results obtained with sera from all of the healthy control subjects, both tuberculin-positive and tuberculin-negative. True positive and false positive fractions were plotted for each antigen at each serum dilution end-point from $<1$ in 20 to 1 in 320 .

\section{Discussion}

It is apparent from our data that patients with active pulmonary tuberculosis develop IgG antibodies to many mycobacterial antigens and that these antibodies can be measured by ELISA techniques. If ELISA is going to be used as a serodiagnostic test, it is important to consider the results achieved with each antigen used in terms of receiver operating characteristics. High specificity is important, because in a situation of low prevalence, false-positive results in the many persons without disease may easily outnumber true positive results in persons with disease.

Nassau et al. (1976) used a filtrate of $M$. tuberculosis in their pioneering studies and 
achieved a sensitivity of $61 \%$ and a specificity of $96 \%$ with serum diluted 1 in 500 . In later studies (Grange et al., 1980; Kardjito and Grange, 1982; Kardjito, Handoyo and Grange, 1982) obtained similar good results with antigen preparations from sonicates of mycobacterial cells, although their data were not reported in sufficient detail to allow calculation of sensitivity and specificity. In the present study $M$. tuberculosis culture filtrate was distinctly inferior to the other, more purified antigens.

PPD has been used in ELISA by several investigators (Tandon et al., 1980; Viljanen, Eskola and Tala, 1982; Zeiss et al., 1982; Kalish et al., 1983; Radin et al., 1983) with good results. It is notable that Kalish et al. (1983), whose study included the largest number of control subjects reported to date in tests with PPD, achieved a sensitivity of $89 \%$ and a specificity of $87 \%$ when an optical density index of $\geqslant 0.30$ was regarded as positive. In our present study, the receiver operating characteristics of ELISA with PPD reflect this favourable experience, although the results were not as good as those with antigen 5 . However, PPD is more readily prepared and more generally available than antigen 5 .

It is notable that our results with $\mathrm{PPD}_{\mathrm{a}}$, prepared from $M$. tuberculosis, were essentially similar to those achieved with $P P D_{k}$, prepared from $M$. kansasi. Neither antigen discriminated between patients with disease due to $M$. tuberculosis and $M$. kansasi. However, both antigens yielded significantly different geometric mean titres between these two infections and disease due to $M$. intracellulare.

In this study, antigen 5 was distinctly superior to the other antigens tested. As seen in fig. 6, at all levels of sensitivity, tests with this antigen produced the more specific result. In our previous work with this antigen (Benjamin and Daniel, 1982), sensitivity was $89 \%$ and specificity $87 \%$ at a 1 in 40 serum dilution; at a 1 in 80 serum dilution these values were $79 \%$ and $92 \%$ respectively. In the present study, ELISA with antigen 5 had a sensitivity of $85 \%$ and a specificity of $87 \%$ at a serum dilution of 1 in 40 , and a sensitivity of $70 \%$ and a specificity of $89 \%$ at a serum dilution of 1 in 80 , based on the results obtained with sera from patients with tuberculosis and from the tuberculinpositive and tuberculin-negative healthy control subjects. Despite our previous observation (Daniel et al., 1979) that antigen 5 is limited in distribution to $M$. tuberculosis and $M$. bovis among 14 species of mycobacteria studied, ELISA with antigen 5 was less effective than ELISA with PPD in discriminating between tuberculous and nontuberculous mycobacterial disease.

In the study of Stroebel et al. (1982), ELISA with antigen 6 was highly specific. That experience was not repeated in the present study. The only other study in which a highly purified antigen was used in ELISA is that of Reggiardo, Vazquez and Schnaper (1980) who used a glycolipid antigen and achieved a sensitivity of $96 \%$ and a specificity of $97 \%$.

In conclusion, ELISA tests performed with antigen 5, antigen 6, $P D_{a}$, and $P P D_{k}$ were capable of distinguishing between patients with mycobacterial disease and control subjects without mycobacterial disease, including patients with sarcoidosis. $\mathrm{PPD}_{\mathrm{a}}$ and $\mathrm{PPD}_{\mathrm{k}}$ ELISA results provided the greatest discrimination between the various mycobacterial diseases. However, the receiver operating characteristics of ELISA with antigen 5 were the most satisfactory when the results obtained with tuberculosis patients were compared with those of tuberculin-positive and tuberculinnegative healthy control subjects. If ELISA is to be applied to the serodiagnosis of tuberculosis in populations or groups of persons suspected of having tuberculosis, 
antigen 5 is probably the best antigen among those studied. The ability to discriminate between individual mycobacterial infections is less important because of the rarity of disease due to mycobacteria other than $M$. tuberculosis.

This work was supported by a grant from Consolidated Biomedical Laboratories, Columbus, OH; by grant AI-15102 from the National Institute for Allergy and Infectious Diseases, Bethesda, MD; and by training grant HL-07288 from the National Heart, Lung, and Blood Institute, Bethesda, MD. We thank Dr C. H. Kirkpatrick of the National Jewish Hospital for the gift of sera from patients with $M$. kansasi and $M$. intracellulare infections.

\section{REFERENCES}

Benjamin R G, Daniel T M 1982 Serodiagnosis of tuberculosis using enzyme linked immunoabsorbent assay (ELISA) of antibody to Mycobacterium tuberculosis antigen 5. American Review of Respiratory Disease 126:1013-1016.

Daniel T M, Anderson P A 1977 The use of immunoabsorbents for the purification of mycobacterial antigens. Journal of Laboratory and Clinical Medicine 90:354-360.

Daniel T M, Anderson P A 1978 The isolation by immunoabsorbent affinity chromatography and physicochemical characterization of Mycobacterium tuberculosis antigen 5. American Review of Respiratory Disease 117:533-539.

Daniel T M, Ellner J J, Todd L S, McCoy D W, Payne V D N, Anderson P A, Bhe F T 1979 Immunobiology and species distribution of Mycobacterium tuberculosis antigen 5. Infection and Immunity 24:77-82.

Daniel T M, Ferguson L E 1970 Purification and characterization of two proteins from culture filtrates of Mycobacterium tuberculosis $\mathrm{H}_{37} \mathrm{Ra}$ strain. Infection and Immunity 1:164-168.

Grange J M, Gibson J, Nassau E, Kardjito T 1980 Enzyme-linked immunosorbent assay (ELISA): A study of antibodies to Mycobacterium tuberculosis in the IgG, IgA and IgM classes in tuberculosis, sarcoidosis and Crohn's disease. Tubercle 61:145-152.

Kalish S B, Radin R C, Phair J B, Levitz D, Zeiss C R, Metzger E 1983 Use of an enzyme-linked immunosorbent assay technique in the differential diagnosis of active pulmonary tuberculosis in humans. Journal of Infectious Diseases 147:523-530.

Kardjito T, Grange J M 1982 Diagnosis of active tuberculosis by immunological methods. 2. Qualitative differences in the dermal response to tuberculin in patients with active pulmonary disease and healthy tuberculin-positive individuals. Tubercle 63:275-278.

Kardjito T, Handoyo T, Grange J M 1982 Diagnosis of active tuberculosis by immunological methods. 1. The effect of tuberculin reactivity and previous $B C G$ vaccination on the antibody levels determined by ELISA. Tubercle 63:269-274.

Ma Y, Daniel T M 1983 Immunochemical analysis of tuberculin purified protein derivative with special reference to United States-Japan antigen 7. Journal of Infectious Diseases 148:500-509.

Metz C E 1978 Basic principles of ROC analysis. Seminar in Nuclear Medicine 8:283-298.

Morrison D F 1976 Multivariate statistical methods, 2nd edn. McGraw-Hill, New York.

Nassau E, Parsons E R, Johnson G D 1976 The detection of antibodies to Mycobacterium tuberculosis by microplate enzyme-linked immunosorbent assay (ELISA). Tubercle 57:67-70.

Radin R C, Zeiss C R, Phair J P 1983 Antibodies to purified protein derivative in different immunoglobulin classes in the diagnosis of tuberculosis in man. International Archives of Allergy and Applied Immunology 70:25-29.

Reggiardo Z, Vazquez E, Schnaper L 1980 ELISA tests for antibodies against mycobacterial glycolipids. Journal of Immunological Methods 34:55-60.

Stroebel A B, Daniel T M, Lau J H K, Leong J C Y, Richardson H 1982 Serological diagnosis of bone and joint tuberculosis by an enzyme-linked immunosorbent assay. Journal of Infectious Diseases 146:280-283.

Tandon A, Saxena R P, Saxena K C, Jamil Z, Gupta A K 1980 Diagnostic potentialities of enzyme-linked immunosorbent assay in tuberculosis using purified tuberculin antigen. Tubercle 61:87-89. 
Viljanen M K, Eskola J, Tala E 1982 Enzyme-linked immunosorbent assay (ELISA) for antibodies to purified protein derivative of tuberculin (PPD). IgM-, IgA-, and IgG-antiPPD antibodies in active pulmonary tuberculosis. European Journal of Respiratory Diseases 63:257-262.

Zeiss G R, Radin R C, Williams J E, Levitz D, Phair J P 1982 Detection of immunoglobulin G antibody to purified protein derivative in patients with tuberculosis by radioimmunoassay and enzyme-linked immunosorbent assay. Journal of Clinical Microbiology 15:93-96. 